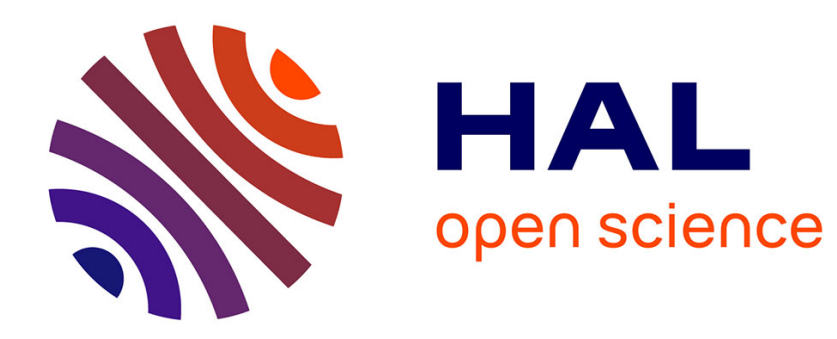

\title{
Planète capitaliste cherche humains disponibles 24/7
}

Agnès Cavet

\section{To cite this version:}

Agnès Cavet. Planète capitaliste cherche humains disponibles 24/7. Lectures, 2014. halshs-01934610

\section{HAL Id: halshs-01934610 \\ https://shs.hal.science/halshs-01934610}

Submitted on 26 Nov 2018

HAL is a multi-disciplinary open access archive for the deposit and dissemination of scientific research documents, whether they are published or not. The documents may come from teaching and research institutions in France or abroad, or from public or private research centers.
L'archive ouverte pluridisciplinaire HAL, est destinée au dépôt et à la diffusion de documents scientifiques de niveau recherche, publiés ou non, émanant des établissements d'enseignement et de recherche français ou étrangers, des laboratoires publics ou privés. 


\section{Planète capitaliste cherche humains disponibles $24 / 7$}

À propos de : Jonathan Crary, 24/7. Le capitalisme à l'assaut du sommeil, Paris, Zones, 2014.

\section{Agnès Cavet}

\section{(2) OpenEdition}

1 Journals

Édition électronique

URL : http://journals.openedition.org/lectures/15039

ISSN : 2116-5289

Éditeur

Centre Max Weber

Ce document vous est offert par Bibliothèque Diderot de Lyon ENS

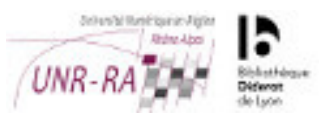

Référence électronique

Agnès Cavet, «Planète capitaliste cherche humains disponibles 24/7 », Lectures [En ligne], Les notes critiques, 2014, mis en ligne le 25 juin 2014, consulté le 26 novembre 2018. URL : http://

journals.openedition.org/lectures/15039

Ce document a été généré automatiquement le 26 novembre 2018.

(c) Lectures - Toute reproduction interdite sans autorisation explicite de la rédaction / Any replication is submitted to the authorization of the editors 


\section{Planète capitaliste cherche humains disponibles $24 / 7$}

À propos de : Jonathan Crary, 24/7. Le capitalisme à l'assaut du sommeil, Paris, Zones, 2014.

\section{Agnès Cavet}

1 À une époque où les sciences de la vie et de la santé donnent l'impression de détenir l'essentiel de la connaissance savante sur le sommeil et ses «troubles", il est fort réjouissant de voir surgir un ouvrage qui propose une lecture socio-politique de ce phénomène. Voilà en effet un petit livre percutant et redoutable, qui met en lumière les menaces suspendues sur ce temps intime - trop souvent impensé - que constitue notre sommeil quotidien. Un temps qui serait aujourd'hui sous l'emprise du néolibéralisme mondialisé, dans lequel la maximisation de l'efficacité et des profits passe par l'imposition du tempo « 24/7 »- vingt-quatre heures sur vingt-quatre, sept jours sur sept.

Richement nourri par ses lectures philosophiques, historiques et sociologiques (Gilles Deleuze et Félix Guattari, Michel Foucault, Karl Marx, John Dewey, Henri Lefebvre, Luc Boltanski et bien d'autres), Jonathan Crary trouve également inspiration dans la littérature, la peinture et le cinéma (Franz Kafka, Italo Calvino, Joseph Wright, Andreï Tarkovski, Chris Marker...). Par cet essai brillant et très accessible, le chercheur new yorkais se positionne moins comme théoricien de l'art - sa spécialité - que comme féroce penseur du monde contemporain. La critique sociale du temps qu'il y développe n'est pas sans évoquer les travaux de Hartmut Rosa sur l'Accélération ${ }^{1}$ - qui pourtant ne sont pas cités. Toutefois, l'essai de Crary ne prétend pas énoncer une théorie mais plutôt entraîner le lecteur, au fil d'un propos d'une grande clarté, dans une réflexion édifiante sur notre vie quotidienne et l'étiolement de notre humanité, en rupture de valeurs sociales. L'« espace de résistance éditoriale » qu'offre le label Zones des éditions de La Découverte sied donc parfaitement à cette entreprise ${ }^{2}$.

L'ouvrage comporte quatre chapitres ; ceux-ci n'étant ni titrés ni clairement structurés, leur fonction n'est-elle pas surtout de rythmer l'écriture de l'auteur tout en modérant l'ardeur du lecteur, le blanc de la page entre deux chapitres invitant à la rêverie et à la 
méditation? Une façon de rompre volontairement la continuité pour rappeler l'importance des temps de pause, de répit et de repos?

\section{Un (ultime ?) grain de sable ${ }^{3}$ dans les rouages du capitalisme}

4 La thèse centrale pourrait se résumer en quelques phrases. Nouveau slogan de notre société capitaliste, le «concept» - au sens marketing du terme - du 24/7 induit une temporalité à la fois statique, redondante et indifférenciée. Elle adresse à l'homme contemporain une injonction au renouvellement incessant de ses besoins de consommation et d'interaction sociale, sans plus que le lieu, le jour ni l'heure n'y oppose de limite. Ce mode de présence au monde 24/7, qui se développe non seulement dans l'espace physique des grandes métropoles de la planète mais aussi et surtout dans le cyberespace, sollicite donc un nouveau modèle d'humain, toujours disponible, connecté, actif, efficace, sans ombre... et bien sûr éveillé. Le besoin naturel de sommeil, qui pousse quotidiennement chacun à se retrancher dans l'espace intime, obscur et sécurisé de la chambre à coucher pour abandonner son corps à un état passif et improductif, apparait alors dépassé - « un truc de losers »-, sans parler de l'inestimable manque à gagner qu'il inflige à l'économie, en empêchant le dormeur de consommer durant ces précieuses heures. Si des besoins élémentaires tels que «la faim, la soif, le désir sexuel et, récemment, le besoin d'amitié ont été convertis en forme marchande ou financiarisée ", le sommeil constitue la dernière poche de résistance au capitalisme puisque, a priori, «l'on ne peut pas en extraire de la valeur» (p. 20). «Temps de cerveau humain » in-

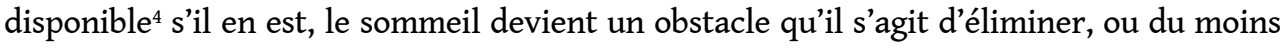
d'assujettir. Si un faisceau d'indices témoigne déjà des entreprises - évoquées plus loin qui visent à libérer l'homme de cette contingence, force est de constater que le citoyen du $\mathrm{XXI}^{\mathrm{e}}$ siècle s'attelle déjà lui-même au travail de sape : le temps de sommeil moyen d'un adulte aurait diminué de plus de trois heures en un siècle; ce temps est aussi érodé par l'insomnie dont souffrent un nombre croissant de nos contemporains; et c'est de plein gré que nous sacrifions encore une part de nos nuits, les yeux rivés sur nos écrans jusqu'à l'épuisement...

5 Les innovations militaires d'un jour se retrouvent bien souvent transposées le (sur)lendemain dans la société civile, sous forme de nouveaux produits. C'est pourquoi il y a lieu d'être attentif aux recherches actuelles du Pentagone, qui visent à émanciper le soldat en opération du besoin de dormir, afin qu'il puisse mener à bien une mission de plusieurs jours sans « défaillance humaine ». Ce fait d'apparence anecdotique constitue avec quelques autres - le point de départ de Crary. Un tel projet n'aurait pu être envisagé si le terreau social n'était déjà prêt à accueillir l'idée fantasmatique d'un homme dégagé de ses exigences corporelles, capable de "profiter " pleinement de ce monde globalisé dont il est parvenu à optimiser le fonctionnement et le rendement 24/7. Autrement dit un homme infiniment répétitif, libéré des "textures rythmiques et périodiques» de sa condition d'humain, un homme foncièrement asocial sous des apparences d'hypersocialité, voué à l'insatisfaction, gaspillant à l'infini, amnésique, sans projet à long terme... à l'exacte image du modèle d'économie hégémonique que l'Occident a mis quelques siècles à parfaire et à imposer à la planète. 


\section{Du sommeil vestige prémoderne de l'expérience humaine...}

6 Mais revenons aux sources du mal. Avec l'avènement du capitalisme, explique Crary, c'est d'abord le travail qui a été coupé des temporalités cycliques liées aux astres (soleil, lune), sur lesquelles reposait l'activité agricole autrefois dominante. C'est donc en premier lieu le temps de travail qui a été homogénéisé, rationnalisé et remodelé sur le mode non-stop les trois huit -, pour passer outre les contraintes sociales et naturelles de l'agent humain. Cependant, le reste de la vie, que l'auteur désigne sous les termes de "vie quotidienne " ou de "quotidien $»^{5}$, est longtemps resté ponctué par le cycle des saisons, les jours de fête, les alternances entre jour et nuit, le rythme des besoins charnels et de leur satisfaction. Ce qui conduit à considérer la vie quotidienne, au cœur de laquelle se trouve le sommeil, comme le site où se concentrent les vestiges prémodernes de l'expérience humaine ${ }^{6}$. C'est seulement après la seconde guerre mondiale que le capitalisme a pu investir des pans importants de la vie quotidienne, dans son espace comme dans son temps, avec l'arrivée dans les foyers de la télévision puis de l'ordinateur personnel, de l'Internet et des divers formats d'objets smart dont notre époque est si friande.

7 Le slogan « $24 / 7$ » tend à la fois, de façon terrifiante, à la « mise au rebut du jour » et à «l'extinction des ténèbres et de l'obscurité ». En théoricien de l'art et de l'esthétique, Crary y décèle un « vaste processus d'incapacitation de l'expérience visuelle ( (p. 45), qui transforme notre façon de percevoir le monde. Il analyse les progrès technologiques et le rythme incessant de production-consommation-obsolescence d'objets électroniques toujours plus performants comme un processus qui colonise à chaque instant le temps, l'attention et l'expérience sensorielle des individus, concourant à les rendre captifs, dociles, malléables et consentants, déconnectés du passé comme de l'avenir - à force d'être connectés à ce temps soi-disant réel, pourrait-on dire. Ces dispositifs technologiques qui, selon Giorgio Agamben ${ }^{7}$, modèlent, contaminent et contrôlent nos existences, ont pour effet de synchroniser et de standardiser l'expérience individuelle à l'échelle mondiale, œuvrant ainsi à la perte des «identités subjectives et singulières ».

Mais les objets technologiques ne sont pas seuls à participer de ce mouvement. En effet, dans le même temps, l'industrie pharmaceutique est parvenue à pathologiser tout une gamme d'états émotionnels (de la timidité aux fluctuations de la libido en passant par la distraction, la tristesse, l'angoisse et bien sûr l'insomnie) pour imposer la consommation de remèdes qui reformatent chimiquement la norme des ressentis individuels tout en procurant un effet artificiel de tranquillisation. Ainsi l'individu contemporain est-il en train de devenir «ce sujet accommodant qui se soumet à toutes sortes d'intrusions biométriques et de surveillances, qui ingère de la nourriture et de l'eau toxiques, et qui vit à proximité de réacteurs nucléaires sans s'en plaindre ", autant de signes de son « abdication absolue de la responsabilité de vivre » (p. 72), estime Crary.

\section{... au sommeil protecteur des valeurs du social}

Quel est le sens profond du sommeil et quelle importance revêt-il pour la vie sociale ? Comme l'ont remarqué de nombreux philosophes depuis le XVII ${ }^{e}$ siècle ${ }^{8}$, le sommeil semble antagonique avec « les opérations de l'esprit et la recherche de la connaissance » 
(p. 21). Il symbolise aussi la passivité et la soumission des «masses endormies ", que seul un « réveil des consciences » pourrait libérer des pouvoirs qui les dominent. La vigilance de l'insomnie apparaît alors à Emmanuel Levinas" comme un "refus de fermer les yeux » sur les injustices et les horreurs du monde ${ }^{10}$.

Mais le sommeil n'est-il qu'un temps d'inaction, donc un temps inutile et impuissant face à ces injustices? Pour Hannah Arendt ${ }^{11}$, notre capacité d'œuvrer à l'intérêt commun dépend au contraire d'un nécessaire équilibre entre l'exposition dans l'espace public et le retrait dans "les ténèbres de la vie cachée ", intime. C'est pourquoi le sommeil ne s'oppose pas à l'action publique mais constitue plutôt une source de régénération des forces de cette action ${ }^{12}$. De plus, "en tant qu'état le plus privé, le plus vulnérable et commun à tous, le sommeil dépend crucialement de la société pour se maintenir » (p. 35). C'est donc cette indispensable vigilance mutuelle sur le repos d'autrui qui fait que «le sommeil représente la durabilité du social» (p. 35), avance Crary, qui voit également un parallèle entre les assauts contre le sommeil et le démantèlement des protections sociales qui caractérise notre époque. D'où l'importance de considérer le sommeil comme «un état qui ne peut pas être instrumentalisé où contrôlé de façon externe » (p. 34-35) et donc comme un noyau de résistance incoercible.

\section{Du rêve freudien privatisé par le désir individuel...}

11 Qu'en est-il plus précisément du rêve ? Jusqu'au XVII siècle, le rêve était considéré - « de façon presque universelle ", selon Crary - comme un composant à part entière à la fois de la vie des individus et de celle des communautés. Mais l'époque moderne s'est attelée à invalider la capacité imaginative ou visionnaire du rêveur, réservant ce pouvoir aux poètes, aux artistes et aux hallucinés. Sigmund Freud ${ }^{13}$ aurait achevé la discréditation du dormeur en réduisant le rêve à l'expression déguisée d'un désir intime refoulé. Le vaste écho qu'a recueilli la théorie freudienne d'interprétation des rêves apparaît à Crary comme «l'indice de la profondeur avec laquelle la primauté du désir et des besoins individuels a pénétré et structuré la compréhension bourgeoise de soi-même depuis le début du XXe siècle » (p. 120).

Un siècle plus tard, Crary récuse cette lecture du rêve, arguant qu'«il serait impossible aujourd'hui d'identifier une aspiration ou un désir individuel qui soit à ce point inavouable qu'il ne puisse être reconnu de manière consciente et satisfait au moins de façon indirecte $»^{14}$; à preuve la profusion de contenus pornographiques accessibles à tous ; à preuve l'engouement que suscitent les émissions de téléréalité, où l'on se bouscule pour porter ses petits secrets au grand jour médiatique. De l'interprétation freudienne, Crary dénonce encore l'étroitesse d'horizon, le rêve se trouvant cantonné à une signification non seulement privée mais également prisonnière du passé ${ }^{15}$ - les premières années de la vie de l'individu. C'est pourquoi il lui préfère la vision d'André Breton, qui réintroduit à la fois la dimension sociale du rêve, sa capacité créatrice et son orientation vers l'avenir. Car pour Breton, le rêve est le véhicule qui assure une circulation et une influence réciproque entre l'activité éveillée et l'état de sommeil, selon le principe des « vases communicants »; collaboration entre action et rêve que le surréaliste voyait déjà propice à « balayer le monde capitaliste $»^{16}$.

Aujourd'hui, les neurosciences considèrent que l'activité onirique entretient une fonction de reprogrammation neuronale essentielle pour «réajuster » l'individu aux évolutions 
quotidiennes de son environnement. Mais si les neurosciences ont peu à dire du contenu même du rêve, elles alimentent l'idée populaire fantasmatique qu'il sera un jour possible de scanner le cerveau pour « enregistrer » les rêves et les (re)visionner à l'état de veille ${ }^{17}$. Ainsi notre époque rêverait-elle de voir dans le rêve un contenu que chacun pourrait détacher de lui-même et bientôt télécharger pour en faire un nouvel objet de consommation et de spectacle, porté sur les réseaux sociaux au crédit de son identité numérique...

\section{... au discrédit des aspirations collectives}

Revenant sur la « révolution mondiale » de 1968 en Europe comme aux États-Unis, dont l'idéologie a gagné également l'Amérique du Sud et l'Asie, Jonathan Crary tente de réhabiliter nombre d'idées, de valeurs et d'expérimentations sociales qui exprimaient déjà le rejet du capitalisme et qui tentaient d'élaborer des solutions alternatives à la société de consommation comme à celle du spectacle dénoncée par Guy Debord ${ }^{18}$. Il montre comment les trente dernières années ont non seulement ruiné les acquis politiques et sociaux des années 1960 mais systématiquement dénaturé, caricaturé et même diabolisé toute ambition sociale égalitaire basée sur une organisation communautaire et sur des valeurs d'entente, de partage et d'entraide, autrement dit les valeurs mêmes que George Herbert Mead considère comme les éléments constitutifs d'une société humaine ${ }^{19}$. De la même façon, sont balayées les approches alternatives du travail et les conceptions du bonheur qui ne se résument pas en une quête de possession et de pouvoir individuels. Ce qui semble aujourd'hui inavouable, ce ne sont plus les désirs individuels que Freud voyait à la source du rêve - et sur lesquels ne plane plus guère de sentiment de culpabilité -, ce sont plutôt les aspirations au refus collectif des injustices, de l'isolement social et de «l'égoïsme obligatoire » que sème le capitalisme néolibéral, estime Crary.

Empruntant à Jean-Paul Sartre ${ }^{20}$ mais aussi à la réalisatrice Chantal Ackerman ${ }^{21}$ l'exemple de la file d'attente, comme situation banale qui met à l'épreuve un conflit entre l'intérêt individuel et l'intérêt social, Crary montre que l'intolérance à l'attente qui caractérise notre $\mathrm{XXI}^{\mathrm{e}}$ siècle entérine pleinement la «négation de la réciprocité » dans les rapports sociaux. Pourtant, «le temps suspendu, improductif de l'attente, chacun son tour, est inséparable de toute forme de coopération et de rapport mutuel » (p. 135). C'est donc bien notre rapport au temps qu'il convient de restaurer si nous ne voulons pas que le néolibéralisme ait définitivement raison de ce qu'il reste de « social » dans notre société. Retrouver la patience d'attendre, savoir marquer des temps d'arrêt et de retrait, refuser l'urgence et, le soir venu, ajourner sans regret les actions du jour pour donner la priorité au repos, telles sont des attitudes qu'il faut sans doute réapprendre pour ménager à nos esprits des interstices de temps dé-connectés du "flux implacable de notre présent " 24/7, propose Crary. Dans ce temps libéré, pourront alors se réinfiltrer la rêverie éveillée et le rêve nocturne, à travers lesquels redécouvrir tout une gamme de perceptions sensorielles ainsi que l'immense pouvoir imaginatif de l'esprit humain. Une imagination à mettre au service de l'invention d'un monde au-delà du capitalisme, un monde tel que l'on aimerait le reconstruire et le partager demain. En concluant ainsi, Crary ne rejoint-il pas Héraclite d'Éphèse qui, cinq siècles avant J.C., affirmait déjà que "les hommes pendant qu'ils dorment travaillent et collaborent au devenir du monde »? 


\section{Euvrer durant nos nuits à rêver l'après-capitalisme}

16

quelque peu radicale - parfois même provocatrice - qui jalonnent son ouvrage. On aurait tort cependant de le résumer à cela. Car ces formules sont les aboutissements auxquels le mène un cheminement à travers les théories philosophiques qu'il croise et discute avec aisance, en donnant toujours au lecteur le sentiment d'aller à l'essentiel. C'est donc cette force d'analyse et de synthèse alliée à la clarté de l'écriture de Crary qui apparaissent comme les qualités maîtresses de son essai. L'attention qu'il apporte aux transformations de la perception constitue également une approche originale et stimulante, dans le prolongement de son précédent ouvrage, Suspensions of Perception: Attention, Spectacle, and Modern Culture ${ }^{22}$. Le lecteur francophone peut encore se réjouir non seulement de l'excellente traduction de Grégoire Chamayou - son éditeur - mais aussi de l'abondance des références françaises exploitées par l'auteur états-unien.

Cependant, après l'éblouissement d'une première lecture, celui qui souhaite approfondir se heurtera peut-être à quelques difficultés, sans doute intrinsèques à la forme de l'essai : le caractère peu structuré de la démonstration, l'auteur procédant souvent par allerretour entre les idées qui apparaissent alors un peu désordonnées ou redondantes; le contraste entre le nombre de grands auteurs convoqués et la relative brièveté des discussions qu'ils suscitent, laissant peu de place aux nuances; et encore, l'absence de mention de certaines sources ${ }^{23}$. Par ailleurs, il nous semble que Crary s'attarde peu sur l'importance de la nuit ${ }^{24}$, qu'il élude en une phrase les complexes relations entre sommeil et mort, et qu'il gagnerait peut-être à s'intéresser davantage aux travaux d'anthropologie sur le sommeil ${ }^{25}$. C'est pourquoi l'on souhaiterait que ce petit essai, tour d'horizon de la question, soit prolongé par un ouvrage peut-être plus académique mais aussi plus systématique et plus approfondi.

18 Le tableau plus noir que la nuit que l'auteur dresse de notre société devrait rencontrer l'adhésion des plus pessimistes ${ }^{26}$. D'autres lecteurs moins fatalistes auront sans doute plus de mal à admettre que l'homme du XXI siècle n'ait plus aucune capacité d'action politique et que la meilleure façon de contrer le capitalisme soit à rechercher dans la réhabilitation du pouvoir visionnaire de rêver. Mais après tout, un homme averti en vaut deux et les intuitions de Crary se fondent sur son vécu de citoyen états-unien, qui capte certains signes alarmants qui ne sont peut-être pas encore parvenus - ou d'une façon plus atténuée - sur le vieux continent européen.

19 Il n'en demeure que ce tonique livre de chevet est à lire et à faire lire largement autour de soi, pour la critique sociale implacable qu'il déroule mais aussi, à notre sens, pour l'occasion qu'il offre de méditer sur la portée philosophique de notre usage du temps; et enfin, pour l'opportunité qu'il donne de comprendre que le sommeil est peut-être le conservatoire le plus précieux de notre humanité archaïque, qui nous relie chacun à toutes les générations d'humains ayant habité cette planète, et qui nous rend responsables envers celles qui viendront de rêver un avenir meilleur pour cette humanité. Prendre la peine d'y réfléchir - avant de s'endormir paisiblement - n'est sûrement pas une perte de temps! 


\section{NOTES}

1. Hartmut Rosa, Accélération. Une critique sociale du temps, La Découverte, coll. "Théorie critique ", 2010. Comptes rendus pour Lectures d'Elodie Wahl : http://lectures.revues.org/990 et de Pascal Descarpes (pour la réédition de 2013) : http://lectures.revues.org/12609. Hartmut Rosa, Aliénation et accélération. Vers une théorie critique de la modernité tardive, La Découverte, coll. "Théorie critique», 2012. Compte rendu de Sébastien Broca pour Lectures: http:// lectures.revues.org/8447.

2. Jonathan Crary a lui-même fondé à New York une maison d'édition indépendante à but non lucratif, Zone books (sans lien avec le label français, malgré la similitude des intitulés), spécialisée dans l'art, les humanités et les sciences sociales.

3. Sans nul doute celui du " marchand » du même nom. Voir la nouvelle "L'homme au sable ", in

E. T. A Hoffmann, Contes nocturnes, Paris, Garnier, coll. « Classiques », 2011 (1817).

4. Pour reprendre la célèbre formule décomplexée de Patrick Le Lay, alors PDG de TF1 : «Ce que nous vendons à Coca-Cola, c'est du temps de cerveau humain disponible » (Les associés d'EIM, Les dirigeants face au changement, Éditions du Huitième jour, 2004).

5. Plus précisément, Crary définit le quotidien comme « une constellation vague des espaces et des temps en dehors de ce qui est organisé autour du travail, de la conformité et du consumérisme » (p. 82).

6. Voir aussi Anson Rabinbach, Le moteur humain. L'énergie, la fatigue et les origines de la modernité, Paris, La Fabrique, 2004. Compte rendu de Jean-Baptiste Marongiu : http://www.lafabrique.fr/ catalogue.php?idArt=62\&idMot=63.

7. Giorgio Agamben, Qu'est-ce qu'un dispositif?, Paris, Payot-Rivages, 2007.

8. L'auteur cite en l'occurrence Descartes, Hume, Locke...

9. Emmanuel Levinas, De l'existence à l'existant, Paris, Vrin, 1986 ; Emmanuel Levinas, Autrement qu'être ou au-delà de l'essence, Paris, Le livre de poche, 1990.

10. Thèse que, nous-semble-t-il, prolonge à sa façon Pierre Pachet dans l'introduction de La force de dormir, Paris, Gallimard, coll. «NRF essais », 1988. Voir aussi Pierre Pachet, Nuits étroitement surveillées, Paris, Gallimard, coll. «NRF essais », 1980.

11. Hannah Arendt, La Condition de l'homme moderne, Paris, Agora/Pocket, 1961.

12. Sur l'approche philosophique, on peut consulter aussi Pierre Carrique, Essai sur la philosophie du sommeil et de la veille, Paris, Gallimard, « NRF », 2002.

13. Sigmund Freud, L'interprétation des rêves, Paris, PUF, 1967 (1899).

14. Crary évoque un autre argument, cette fois rétrospectif, considérant que « la notion même de "désirs individuels" était sans doute dénuée de sens pendant la majeure partie de l'histoire de l'humanité » (p. 120).

15. Deux limites que récusait Carl Gustav Young, qui d'ailleurs n'admettait pas non plus la différence entre le contenu manifeste et le contenu latent du rêve, sur laquelle repose la théorie freudienne.

16. André Breton, Les Vases communicants, Paris, Gallimard, 1955 (1932), p. 166. Crary rappelle, non sans malice, "l'incompréhension condescendante » que Freud a réservée à cet ouvrage, envoyé par Breton.

17. Un tel fantasme hight tech était par exemple mis en scène dans un film de Wim Wenders, Jusqu'au bout du monde, 1991.

18. Guy Debord, La société du spectacle, Paris, Gallimard, coll. « Folio », 2002 (1967). 
19. George Herbert Mead, L'esprit, le soi et la société, Paris, PUF, 2006. Voir aussi Alexis Cukier, Éva Debray (dir.), La théorie sociale de G.H. Mead. Études critiques et traductions inédites, Lormont, Le Bord de l'eau, coll. "La bibliothèque du Mauss », 2014. Compte rendu de Christian Guinchard pour Lectures : http://lectures.revues.org/14886.

20. Jean-Paul Sartre, Critique de la raison dialectique, Paris, Gallimard, 1960.

21. Chantal Ackermann, D'Est. Au bord de la fiction, Paris, Éditions du Jeu de Paume, 1995. Ouvrage qui fait suite à son film D'Est, 1993.

22. Jonathan Crary, Suspensions of Perception: Attention, Spectacle and Modern Culture, Cambridge (Mass.), MIT, 2000.

23. Impossible, par exemple, de savoir d'où l'auteur tient l'information selon laquelle un adulte occidental dormait dix heures par jour au début du XX $\mathrm{X}^{\mathrm{e}}$ siècle. Crary ne cite pas non plus ses sources lorsqu'il affirme que « des recherches récentes ont montré que le nombre de personnes qui se lèvent la nuit pour consulter leurs messages électroniques ou accéder à leurs données est en train de croître de façon exponentielle » (p. 22-23).

24. Voir par exemple Anne Cauquelin, La ville la nuit, Paris, PUF, coll. "La politique éclatée », 1977.

25. Citons par exemple: Guillaume Garnier, L'oubli des peines: une histoire du sommeil, 1700-1850, Rennes, PUR, 2013 ; Jacqueline Carroy, Nuits savantes. Une histoire des rêves (1800-1945), Paris, Éditions de l'ÉHÉSS, coll. «En temps et lieu », 2012, compte rendu de Guillaume Garnier : http:// rh19.revues.org/4636 ; Sophie Jama, Rêve et cultures, Montréal, « petite collection Liber », 2009 ; Sophie Jama, Anthropologie du rêve, Paris, PUF, 1997 ; Gérard Ermisse (dir.), " Rêver », Terrain, n - 26, mars 1996 ; Jean Duvignaud, Françoise Duvignaud et Alain Corbin, La banque des rêves. Essai d'anthropologie du rêveur contemporain, Paris, Payot, 1979 ; Pascal Dibie, Ethnologie de la chambre à coucher, Paris, Grasset, coll. «Biblio essais », 1991 ; Agnès Cavet, « Sans dormir », in Alain Renaud et Lucette Savier (dir.), Dormir. L'énigme de chaque nuit, Paris, Autrement, 1991; et encore les numéros 7 (1983) et 14 (1985) de la revue Corps écrit, consacrés respectivement au sommeil et à la nuit.

26. On ne s'étonne donc pas de trouver un article de Jonathan Crary en bonne place au sommaire du Monde diplomatique de ce mois de juin 2014, dans lequel il résume les grandes lignes de son essai : « À l'assaut du sommeil », http://www.monde-diplomatique.fr/2014/06/CRARY/50520.

\section{AUTEUR}

\section{AGNÈS CAVET}

Chargée de production éditoriale, ENS de Lyon. 